\title{
CdS versus ZnSnO buffer layers for a CIGS solar cell: a depth-resolved analysis using the muon probe
}

\author{
Eduardo Ribeiro ${ }^{1,}$, Helena V. Alberto ${ }^{1, *}$, Rui C. Vilão ${ }^{1}$, João M. Gil ${ }^{1}$, Alois Weidinger ${ }^{2}$, \\ Pedro M. P. Salomé ${ }^{3,4}$, Thomas Prokscha ${ }^{5}$, Andreas Suter $^{5}$, and Zaher Salman ${ }^{5}$, \\ ${ }^{1}$ CFisUC, Department of Physics, University of Coimbra, R. Larga, P-3004-516 Coimbra, Portugal \\ ${ }^{2}$ Helmholtz-Zentrum Berlin für Materialien und Energie, 14109 Berlin, Germany \\ ${ }^{3}$ International Iberian Nanotechnology Laboratory, 4715-330 Braga, Portugal \\ ${ }^{4}$ Department of Physics, University of Aveiro, 3810-193 Aveiro, Portugal \\ ${ }^{5}$ Laboratory for Muon Spin Spectroscopy, Paul Scherrer Institut, CH-5232 Villigen PSI, Switzerland
}

\begin{abstract}
The influence of a buffer layer in the surface of a $\mathrm{Cu}(\mathrm{In}, \mathrm{Ga}) \mathrm{Se}_{2}$ (CIGS) solar cell material is studied using implanted positive muons as a probe. A depth resolved analysis of the muon data suggests that both $\mathrm{CdS}$ and $\mathrm{ZnSnO}$ reduce the width of a defect layer present at the CIGS surface to about half its original value. Additionaly, CdS is able to reduce the intensity of the disturbance in the defected region, possibly due to a surface reconstrution in CIGS.
\end{abstract}

\section{Introduction}

Thin film solar cells using $\mathrm{Cu}(\mathrm{In}, \mathrm{Ga}) \mathrm{Se}_{2}$ (CIGS) as the p-type absorber achieved recently $23.3 \%$ solar cell efficiency[1], the highest value among multi-crystalline solar cells. CdS has been the most widely used n-type buffer layer for CIGS solar cell, but has the important disadvantage of containing a toxic element, the cadmium. The material $\mathrm{ZnSnO}$ was shown to constitute a good alternative as a Cd-free buffer layer in CIGS, exhibiting a similar performance to CdS [2, 3].

A previous study [4] using implanted positive muons with energies in the range 2-24 keV has shown that the formation of the final bound configuration of the muon is sensitive to local barriers and that the spatial extent of the decrease in formation probability of the muon state can be used as a measure of the width of a defective region ocurring at the p-n interface.

Recently, a new method of analysis of the energy-dependent muon data was developed, allowing a depth-resolved analysis of all the experimental parameters associated with the muon signal [5]. This paper presents the results obtained for low-energy muon data for a CIGS film and two junctions, CdS/CIGS and $\mathrm{ZnSnO} / \mathrm{CIGS}$ reanalysed using this new method. It is shown that this depth-resolved analysis provides new insights on the microscopic processes ocurring during the formation of the buffer/absorber interface in a CIGS based solar cell.

\section{Experimental Details and Data Analysis}

Two different heterostructures, CdS/CIGS and ZnSnO/CIGS, were produced in the Ångström Solar Center, Uppsala University, Sweden, from one single CIGS film, using a test pilot

\footnotetext{
*e-mail: lena@uc.pt
} 
tool for module production. Extensive details on the sample preparation were reported in a previous publication [6]. The thickness of the n-type layer was $50 \mathrm{~nm}$ for CdS and $20 \mathrm{~nm}$ for $\mathrm{ZnSnO}$. The single CIGS film was obtained by removing the CdS layer from the CdS/CIGS sample by etching with a solution $10 \%(\mathrm{~V} / \mathrm{V})$ of $\mathrm{HCl}$ in $\mathrm{H}_{2} \mathrm{O}$, dried with a $\mathrm{N}_{2}$ blow drier and immediately moved into a $\mathrm{N}_{2}$ atmosphere to avoid surface oxygenation. It was mounted under continuous $\mathrm{N}_{2}$ flow in a sample holder and measured in vacuum.

The muon spin spectroscopy ( $\mu \mathrm{SR})$ measurements were performed at the $\mu \mathrm{E} 4$ beam line [7] of the Swiss Muon Source, Paul Scherrer Institut, Switzerland, using the low energy muons (LEM) instrument. Positive muons were implanted in the samples in the presence of an external magnetic field $\mathrm{B}=10 \mathrm{mT}$, using transverse field (TF) geometry at a fixed temperature of $\mathrm{T}=50 \mathrm{~K}$ and variable implantation energy, in order to perform depth dependent studies, as illustrated in Fig.1.
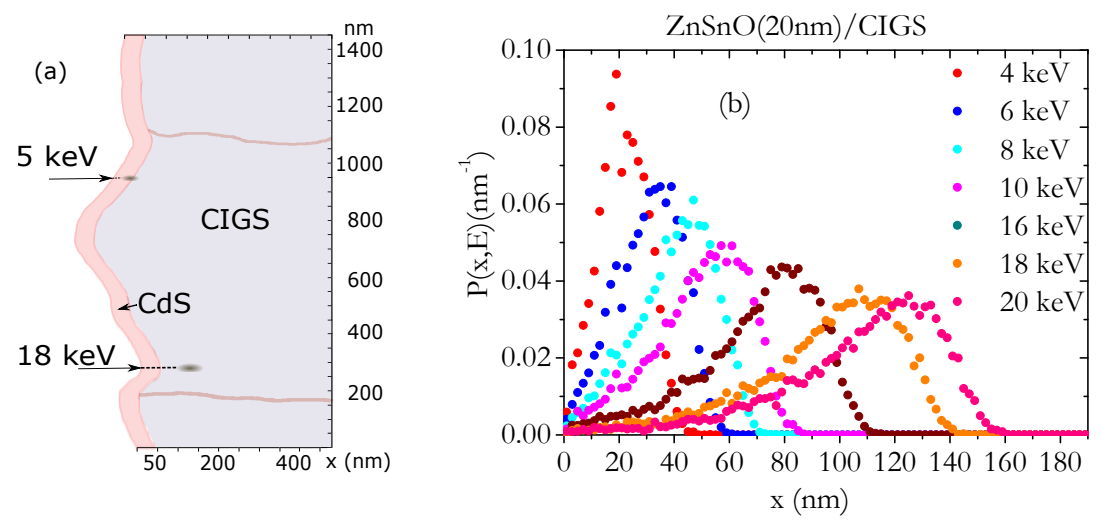

Figure 1. (a): Illustration of the change in implantation depth for two different muon implantation energies, in a CdS/CIGS heterostructure. The drawing is based on a Transmission Electron Microscopy picture. (b): Monte Carlo simulations of the probability per unit lenght, $P(x, E)$ that a muon implanted with energy $E$ stops at a depth $x$ in a $\mathrm{ZnSnO}(20 \mathrm{~nm}) / \mathrm{CIGS}$ junction. The different curves correspond to different muon implantation energies.

The time dependent $\mu \mathrm{SR}$ data corresponds to muons forming a diamagnetic state and was fitted to a Gaussian-damped oscillation at the muon Larmor frequency. The diamagnetic signal amplitude $A_{\text {dia }}$ was converted to the corresponding diamagnetic fraction, $f_{\text {dia }}$, using a silver calibration and a sample size correction. The diamagnetic fraction was found to be smaller than unity for most of the measurements. This indicates that the muons experience a strong interaction during their implantation stage, reducing the formation probability of the final bound configuration [4, 8-13].

From the fitting of the muon time spectra, the diamagnetic fraction and the Gaussian relaxation rate were obtained as a function of the muon implantation energy, $f_{\mathrm{dia}}(E)$ and $\sigma(E)$. Figure 1(b), illustrates the muon stopping distribution in a $\mathrm{ZnSnO} / \mathrm{CIGS}$ sample as a function of sample depth, $x$, as obtained by Monte Carlo simulation, using the TRIM.SP code $[14,15]$. Since the muon stopping region extends over a region of several tens of nanometers, the energy dependence of the relevant $\mu$ SR parameters cannot be interpreted directly as a depth dependence. Therefore, depth dependent functions were inferred using the method described in reference [5] : the functions $f_{\text {dia }}(x)$ and $\sigma(x)$ were assumed to have a steplike shape with adjustable parameters; $f_{\text {dia }}(E)$ and $\sigma(E)$ functions were then calculated by weighting $f_{\mathrm{dia}}(x)$ and $\sigma(x)$ with the corresponding muon stopping probabilities, obtained by 

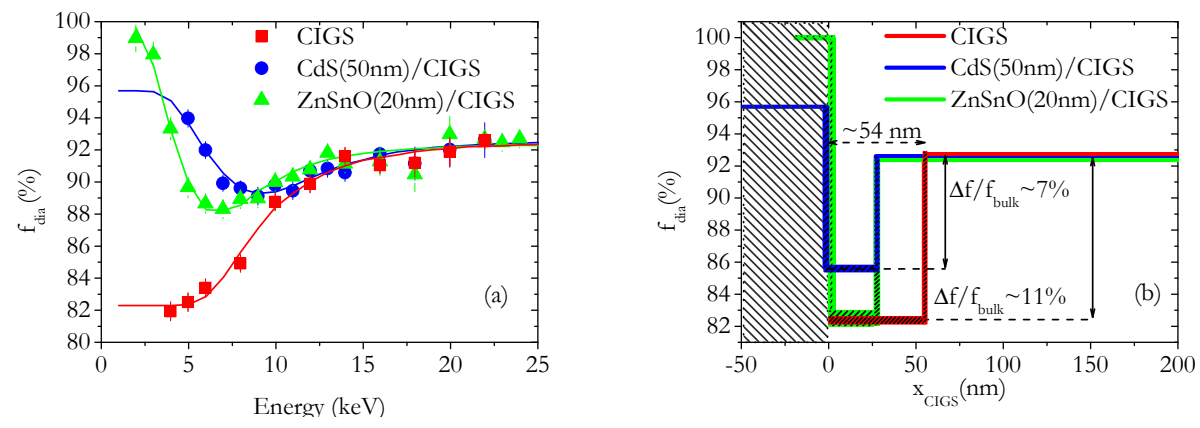

Figure 2. (a) : Diamagnetic fraction as a function of muon implantation energy, $f_{\mathrm{dia}}(E)$, at $50 \mathrm{~K}$ for $\mathrm{CdS} / \mathrm{CIGS}, \mathrm{ZnSnO} / \mathrm{CIGS}$ and CIGS samples. The symbols are the experimental data points. The full curves are the predicted behaviour of the parameter assuming a depth dependence as shown in the corresponding graph at right. (b): Diamagnetic fraction as a function of the distance to the surface of CIGS, $f_{\text {dia }}\left(x_{\text {CIGS }}\right)$. The thickness of the lines indicate the uncertainties in the parameters of $f_{\text {dia }}\left(x_{\text {CIGS }}\right)$, namely the position and depth of the wells.

numerical integration of the muon stopping probability per unit length, $P(x, E)$ (see Fig.1(b)); the experimental data points were then fitted to the calculated $f_{\text {dia }}(E)$ and $\sigma(E)$, in order to optimize the parameters for $f_{\text {dia }}(x)$ and $\sigma(x)$. The thickness of the lines in the plots of the functions $f_{\text {dia }}(x)$ and $\sigma(x)$ (see Fig.2(b) and Fig.3(b)) are a measure of the uncertainties in the parameters, as obtained in this fitting procedure.

\section{Results and Discussion}

Figure 2 shows the diamagnetic fraction as a function of muon implantation energy for the three different samples, CdS/CIGS, ZnSnO/CIGS and the CIGS film at $50 \mathrm{~K}$ and under an external magnetic field $\mathrm{B}=10 \mathrm{mT}$, together with the corresponding functions $f_{\text {dia }}\left(x_{\mathrm{CIGS}}\right)$, where $x_{\text {CIGS }}$ is defined as the distance to the CIGS surface.

It is evident from Fig. 2(b) that the fraction of the muon diamagnetic state for the bare CIGS film is about $11 \%$ smaller at the surface when compared with its bulk value, within a region which is 54(1) $\mathrm{nm}$ wide. The diamagnetic fraction in CIGS is a measure of the formation probability of the final bound configuration of the muon in the lattice and is sensitive to local barriers, such as defects, that may hinder its formation. There are numerous reports that the surface of CIGS is a defect-rich region, which has a slightly different composition from the bulk, namely a smaller $\mathrm{Cu}$ content [16-18]. We attribute the dip in the diamagnetic fraction as due to this surface defect layer (SDL) and the observed spatial extent of the dip $(\sim 54 \mathrm{~nm})$ as a measure of the SDL thickness in the bare CIGS film.

Figure 2(b) also shows that when a top layer of $\mathrm{ZnSnO}$ or $\mathrm{CdS}$ is added, the width of the dip at the CIGS surface is reduced to around half (25 to $28 \mathrm{~nm}$ ); in both samples, the recovery of the diamagnetic fraction to the bulk value clearly occurs in the inner part of CIGS. A possible explanation is that this reduction is due to the built-in electric field at the p-n interface. It has been suggested that the Cu-depletion at the CIGS surface is due to $\mathrm{Cu}$ electro-diffusion due to positive charges at CIGS surface [18]. The formation of a depleted, negatively charged region in CIGS when $\mathrm{CdS}$ or $\mathrm{ZnSnO}$ is added may cause a diffusion of $\mathrm{Cu}$ from the bulk towards the surface, causing a healing in the inner part of the defect layer. 

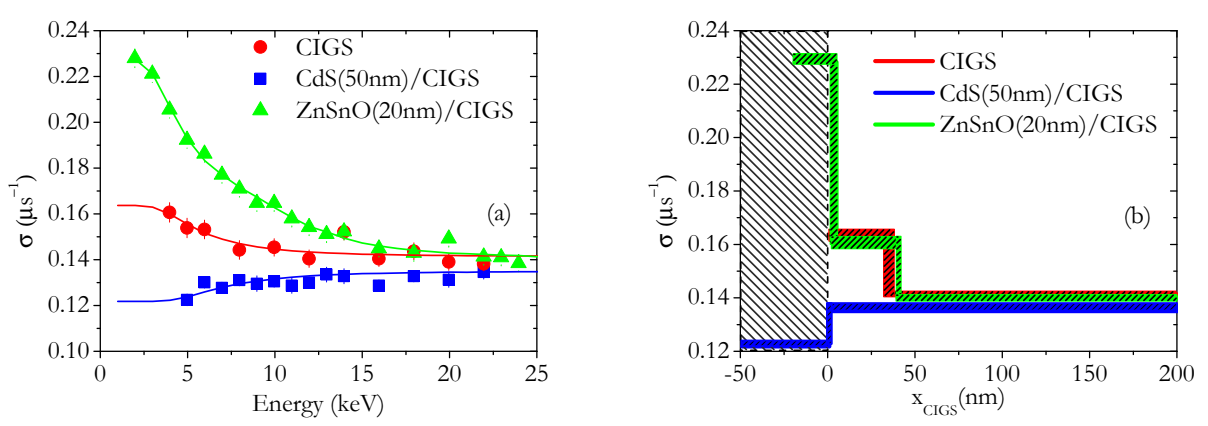

Figure 3. (a) : Muon spin relaxation as a function of muon implantation energy, $\sigma(E)$, at $50 \mathrm{~K}$ for $\mathrm{CdS} / \mathrm{CIGS}, \mathrm{ZnSnO} / \mathrm{CIGS}$ and CIGS samples. The full curves are the predicted behaviour of the parameter assuming a depth dependence as shown in the corresponding graph at right. (b): muon spin relaxation as a function of the distance to the CIGS layer, $\sigma\left(x_{\mathrm{CIGS}}\right)$ for the three samples. The thickness of the lines is a measure of the uncertainties in the parameters of the $\sigma\left(x_{\mathrm{CIGS}}\right)$ function.

Additionally, Fig. 2(b) shows that the depth of the diamagnetic dip in the CIGS region is also reduced for the CdS/CIGS sample (from 11\% in CIGS film to 7\% in CdS/CIGS) but a similar reduction is not observed for the $\mathrm{ZnSnO} / \mathrm{CIGS}$ sample. Therefore CdS causes changes both at the surface and inner part of the SDL in CIGS, whereas $\mathrm{ZnSnO}$ only affects the SDL inner region.

Figure 3 shows that in the CIGS film there is a surface region with a spatial extent of $\sim 35 \mathrm{~nm}$, where the muon spin relaxation is $\sim 14 \%$ larger than in bulk. The increase of the muon spin relaxation at the CIGS surface suggests a change in the muon environment in its final state configuration, caused either by structural disorder, defects, or electron capture. This region at the CIGS surface where the muon suffers a stronger relaxation is still present when $\mathrm{ZnSnO}$ is added on top, but disappears if the top layer is CdS. This suggests again that CdS causes a surface reconstruction of CIGS surface, close to the p-n interface, but that does not occur for $\mathrm{ZnSnO}$. These results are consistent with evidences that when a layer of $\mathrm{CdS}$ is added on top of CIGS there is a inter-diffusion of ions at the interface, but no such intermixing is found when the top layer is $\mathrm{ZnSnO}[3,19]$.

\section{Conclusions}

In conclusion, whereas the diamagnetic muon fraction is sensitive to the barrier for the formation of the final muon state, the muon spin relaxation is sensitive to the muon environment in its final configuration. The combination of the information from both parameters, obtained by a depth-resolved analysis of the muon data, suggests that there is a surface defect layer in CIGS material with a spatial extent of around $54 \mathrm{~nm}$ and that the width of this region is reduced to about half its original value when either a $\mathrm{CdS}$ or $\mathrm{ZnSnO}$ layer is added. However, unlike $\mathrm{ZnSnO}, \mathrm{CdS}$ is also able to induce a surface reconstrution of CIGS, reducing the intensity of the disturbance at the CIGS surface.

\section{Acknowledgement}

This work is based on experiments performed at the Swiss Muon Source $(\mathrm{S} \mu \mathrm{S})$, Paul Scherrer Institute, Villigen, Switzerland. This work was supported with funds from the Portuguese National Budget 
through Fundação para a Ciência e Tecnologia/MCTES and also from the European Regional Development Fund (ERDF) through the Competitiveness and Internationalisation Operational Programa (PTCOMPETE 2020), under projects UID/FIS/04564/2016 and PTDC/FISMAC/29696/2017.

\section{References}

[1] M.A. Green, E.D. Dunlop, D.H. Levi, J. Hohl-Ebinger, M. Yoshita, A.W.Y. Ho-Baillie, Progress in Photovoltaics: Research and Applications 27 (2019)

[2] J. Lindahl, J.T. Wätjen, A. Hultqvist, T. Ericson, M. Edoff, T. Törndahl, Progress in Photovoltaics: Research and Applications 21, 1588 (2013)

[3] P.M.P. Salomé, R. Ribeiro-Andrade, J.P. Teixeira, J. Keller, T. Törndahl, N. Nicoara, M. Edoff, J.C. González, J.P. Leitão, S. Sadewasser, IEEE Journal of Photovoltaics 7, 858 (2017)

[4] H.V. Alberto, R.C. Vilão, R.B.L. Vieira, J.M. Gil, A. Weidinger, M.G. Sousa, J.P. Teixeira, A.F. da Cunha, J.P. Leitão, P.M.P. Salomé et al., Phys. Rev. Materials 2, 025402 (2018)

[5] A.F.A. Simões, H.V. Alberto, R.C. Vilão, J.M. Gil, J.M.V. Cunha, M.A. Curado, P.M.P. Salomé, T. Prokscha, A. Suter, Z. Salman, submitted to Review of Scientific Instruments (2019)

[6] P.M.P. Salomé, J.P. Teixeira, J. Keller, T. Törndahl, S. Sadewasser, J.P. Leitão, IEEE Journal of Photovoltaics 7, 670 (2017)

[7] T. Prokscha, E. Morenzoni, K. Deiters, F. Foroughi, D. George, R. Kobler, A. Suter, V. Vrankovic, Nuclear Instruments and Methods in Physics Research Section A: Accelerators, Spectrometers, Detectors and Associated Equipment 595, 317 (2008)

[8] R.C. Vilão, R.B.L. Vieira, H.V. Alberto, J.M. Gil, A. Weidinger, Phys. Rev. B 96, 195205 (2017)

[9] R.C. Vilão, R.B.L. Vieira, H.V. Alberto, J.M. Gil, A. Weidinger, R.L. Lichti, P.W. Mengyan, B.B. Baker, J.S. Lord, Phys. Rev. B 98, 115201 (2018)

[10] R.C. Vilão, H.V. Alberto, J.M. Gil, A. Weidinger, Phys. Rev. B 99, 195206 (2019)

[11] H.V. Alberto, A. Weidinger, R.C. Vilão, J.P. Duarte, J.M. Gil, J.S. Lord, S.F.J. Cox, Phys. Rev. B 81, 245205 (2010)

[12] H.V. Alberto, R.C. Vilão, J.P. Duarte, J.M. Gil, A. Weidinger, J.S. Lord, S.F.J. Cox, Phys. Rev. B 86, 035203 (2012)

[13] H.V. Alberto, R.C. Vilão, J.M. Gil, J. Piroto Duarte, R.B.L. Vieira, A. Weidinger, J.P. Leitão, A.F. da Cunha, M. Sousa, J.P. Teixeira et al., Journal of Physics: Conference Series 551, 012045 (2014)

[14] E. Morenzoni, H. Glückler, T. Prokscha, R. Khasanov, H. Luetkens, M. Birke, E. Forgan, C. Niedermayer, M. Pleines, Nuclear Instruments and Methods in Physics Research Section B: Beam Interactions with Materials and Atoms 192, 254 (2002)

[15] W. Eckstein, Computer Simulations of Ion-Solid Interactions (Springer, Berlin, Heidelberg, New York, 1991)

[16] D. Liao, A. Rockett, Applied Physics Letters 82, 2829 (2003)

[17] Y. Yan, K.M. Jones, J. Abushama, M. Young, S. Asher, M.M. Al-Jassim, R. Noufi, Applied Physics Letters 81, 1008 (2002), https: //doi .org/10. 1063/1. 1498499

[18] Herberholz, R., Rau, U., Schock, H. W., Haalboom, T., Gödecke, T., Ernst, F., Beilharz, C., Benz, K. W., Cahen, D., Eur. Phys. J. AP 6, 131 (1999)

[19] P.M.P. Salomé, J. Keller, T. Törndahl, J.P. Teixeira, N. Nicoara, R.R. Andrade, D.G. Stroppa, J.C. González, M. Edoff, J.P. Leitão et al., Solar Energy Materials and Solar Cells 159, 272 (2017) 